\title{
Chemotherapy With or Without Anti-EGFR Agents in Left- and Right-Sided Metastatic Colorectal Cancer: An Updated Meta-Analysis
}

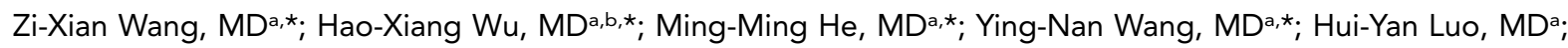
Pei-Rong Ding, MD, PhDc; Dan Xie, MD, PhDd; Gong Chen, MD, PhD c,t; Yu-Hong Li, MD, PhD ${ }^{a, t}$;

Feng Wang, MD, PhD ${ }^{\mathrm{a}, t}$; and Rui-Hua $\mathrm{Xu}, \mathrm{MD}, \mathrm{PhD}^{\mathrm{a}, \mathrm{t}}$

\section{ABSTRACT}

Background: Previous meta-analyses have suggested primary tumor location as a predictive factor for efficacy of anti-epidermal growth factor receptor (EGFR) therapies in patients with metastatic colorectal cancer (mCRC). However, the recent phase III TAILOR trial addressing this issue was not included in those analyses. This meta-analysis incorporated data from the TAILOR trial to evaluate the efficacy of chemotherapy plus anti-EGFR agents (cetuximab [Cet] or panitumumab [Pani]) versus chemotherapy alone for RAS wild-type (wt) right- and left-sided mCRC. Patients and Methods: A PubMed-based literature search was conducted to identify randomized controlled trials (RCTs) studying the additional efficacy of Cet/Pani in combination with chemotherapy versus chemotherapy alone in RAS wt left- and right-sided $\mathrm{mCRC}$. Study-level pooled analyses of hazard ratios (HRs) for overall survival (OS) and progression-free survival (PFS) and odds ratios (ORs) for objective response rate (ORR) were performed. Results: Three first-line RCTs (CRYSTAL, PRIME, and TAILOR) and one secondline RCT (20050181) were included. Significant OS benefits from Cet/ Pani were observed in the left-sided ( $\mathrm{HR}, 0.76$; $95 \% \mathrm{Cl}, 0.66-0.86)$ but not right-sided subgroups ( $\mathrm{HR}, 0.99 ; 95 \% \mathrm{Cl}, 0.78-1.27)$. However, the addition of Cet/Pani to chemotherapy significantly improved PFS and ORR in both the left-sided (HR, 0.70; 95\% Cl, 0.57-0.86, and OR, $3.28 ; 95 \% \mathrm{Cl}, 1.95-5.51$, respectively) and right-sided subgroups (HR, $0.76 ; 95 \% \mathrm{Cl}, 0.59-0.99$, and $\mathrm{OR}, 1.78 ; 95 \% \mathrm{Cl}, 1.08-2.93$, respectively). Conclusions: The addition of Cet/Pani to chemotherapy significantly benefits PFS and ORR in patients with RAS wt right-sided $\mathrm{mCRC}$, indicating that anti-EGFR therapies may remain an option for selected patients.

J Natl Compr Canc Netw 2019;17(7):805-811 doi: 10.6004/jnccn.2018.7279

\footnotetext{
aDepartment of Medical Oncology, Sun Yat-sen University Cancer Center, State Key Laboratory of Oncology in South China, Collaborative Innovation Center for Cancer Medicine; ${ }^{b}$ Faculty of Medical Sciences, Sun Yat-sen University; and Departments of ${ }^{c}$ Colorectal Surgery, and ${ }^{d}$ Pathology, Sun Yat-sen University Cancer Center, State Key Laboratory of Oncology in South China, Collaborative Innovation Center for Cancer Medicine, Guangzhou, China.

*Authors contributed equally to this study.

${ }^{\dagger}$ Joint senior authors.
}

\section{Background}

Colorectal cancer (CRC) is the third most common malignant disease affecting both men and women in the Asia-Pacific region. ${ }^{1}$ Several studies have reported heterogeneity in developmental, microbial, gut floral, molecular, genetic, and clinical outcomes of left- and right-sided CRC tumors. ${ }^{2-7}$ These differing characteristics are associated with differential prognosis, and patients with right-sided CRC are reported to have a worse prognosis. ${ }^{8,9}$

A recent meta-analysis including 14 prospective studies in metastatic CRC (mCRC) reported a $56 \%$ increase in mortality in right-sided compared with leftsided tumors, ${ }^{10}$ indicating the prognostic relevance of primary tumor location (PTL). In addition, several post hoc analyses of randomized controlled trials (RCTs) and retrospective studies have suggested PTL to be a predictive factor for treatment benefits, especially in patients treated with targeted therapies. ${ }^{1-16} \mathrm{~A}$ recent pooled analysis of 6 first- or second-line RCTs evaluating the efficacy of chemotherapy + cetuximab (Cet) or panitumumab (Pani) versus chemotherapy \pm bevacizumab (Bev) reported significant improvement in overall survival (OS), progression-free survival (PFS), and objective response rate (ORR) with chemotherapy + Cet/Pani in patients with $R A S$ wild-type (wt) left-sided tumors, but no significant survival benefits in those with $R A S$ wt right-sided tumors. ${ }^{17}$ In addition, subgroup analysis with Bev in the control arm showed significantly worse PFS and numerically worse OS with chemotherapy + Cet/Pani in patients with RAS wt right-sided mCRC. ${ }^{17}$ Because of these findings, Bev is increasingly being considered the preferred targeted agent in patients with right-sided tumors. ${ }^{18}$

The choice between chemotherapy + Cet/Pani and chemotherapy alone nonetheless remains clinically relevant to patients with $R A S$ wt right-sided mCRC when they are not candidates for Bev therapy. Prior metaanalyses of trials investigating chemotherapy + Cet/Pani 
versus chemotherapy alone failed to show significant improvements in OS, PFS, or ORR with the addition of Cet/Pani in these tumors, ${ }^{10,17}$ but these studies may have had inadequate sample size and statistical power. The recent Chinese phase III TAILOR trial, which investigated the efficacy of chemotherapy + Cet versus chemotherapy alone in the RAS wt population, was not included in the previous analyses. ${ }^{19}$ By including all the relevant RCTs, our meta-analysis aimed to reevaluate the efficacy of chemotherapy plus anti-epidermal growth factor receptor (EGFR) agents (Cet/Pani) compared with chemotherapy alone in relation to PTL in patients with RAS wt mCRC.

\section{Patients and Methods}

This meta-analysis was reported in accordance with PRISMA guidelines. ${ }^{20}$

\section{Study Selection and Data Extraction}

A PubMed-based literature search through the end of December 2017 was performed using the following search or MeSH terms: metastatic colorectal cancer, primary tumor location, sidedness, left-sided tumor, and right-sided tumor. In addition, we conducted searches of the major oncology congress websites, including ASCO and ESMO. After screening titles and abstracts, potentially relevant studies were retrieved and assessed for eligibility criteria. Reference lists of the included publications were manually checked to identify additional potential studies.

Studies that met the following criteria were eligible for the quantitative analysis: (1) RCTs that reported RAS wt (KRAS/NRAS exon 2-4 wt) mCRC; (2) studies that enrolled patients treated with either chemotherapy + anti-EGFR agents (Cet/Pani) or chemotherapy alone; and (3) studies that reported treatment outcomes (OS, PFS, and ORR) as HRs and odds ratios (ORs), with stratification for PTL (left vs right). The most recent and complete publications were included when duplicate data or subgroup analysis of the same study was found.

Relevant data were extracted by 2 independent investigators (Z.X. Wang, H.X. Wu) and verified by a third investigator (M.M. He). Any discrepancies among investigators were resolved through consensus.

\section{Statistical Analyses}

HRs and ORs with 95\% CIs in the literature were synthesized to obtain overall treatment effects based on PTL (left vs right). Potential heterogeneity among the studies was determined using Cochran's $Q$ statistic and $I^{2}$ statistic. In the presence of significant heterogeneity $(P<.10$ or $I^{2}>50 \%$ ), random-effects meta-analytic models were used to calculate the pooled HRs and ORs. Otherwise, the analysis was performed using the fixed-effects models.
Multivariate random-effects meta-regression models were used to evaluate the interaction effect between PTL and treatment with Cet/Pani after adjustment for the antiEGFR agent, chemotherapy backbone, and treatment line. All $P$ values were 2 -sided. Statistical analyses were performed using R 3.4.1 (The R Foundation) with the package "metaphor."

\section{Results}

\section{Study Characteristics}

The search yielded a total of 1,252 potentially relevant studies, of which 4 RCTs ultimately fulfilled the study criteria (CRYSTAL, ${ }^{8,21}$ PRIME, ${ }^{11,22}$ TAILOR, ${ }^{19}$ and $20050181^{23,24}$; ClinicalTrials.gov identifiers: NCT00154102, NCT00364013, NCT01228734, and NCT00339183, respectively) (Figure 1). Baseline characteristics of the included trials are summarized in Table 1. A total of 1,539 patients were included, of which 325 were diagnosed with right-sided $R A S$ wt mCRC and 1,214 with left-sided mCRC (Table 1). In all 4 trials, primary tumors originating in the cecum, appendix, ascending colon, hepatic flexure, and transverse colon were classified as right-sided tumors.

\section{Effect of PTL on OS Benefits With Anti-EGFR Agents}

A significant improvement in OS was observed in patients with left-sided $R A S$ wt tumors treated with chemotherapy + Cet/Pani compared with chemotherapy alone (HR, 0.76; 95\% CI, 0.66-0.86; $P<.0001$; Figure $2 \mathrm{~A}$ ), without significant evidence for between-study heterogeneity $(P=.150$; $\left.I^{2}=43.7 \%\right)$. However, addition of Cet/Pani to chemotherapy provided no OS benefits in patients with $R A S \mathrm{wt}$ right-sided tumors (HR, 0.99; 95\% CI, 0.78-1.27; $P=.945$; $P_{\text {heterogeneity }}=.860 ; I^{2}=0 \%$; Figure $2 \mathrm{~A}$ ). After adjustment for anti-EGFR agent, chemotherapy backbone, and treatment line, the treatment effect of Cet/Pani on OS differed significantly according to PTL $\left(P_{\text {interaction }}=.044\right.$; Table 2).

\section{Effect of PTL on PFS Benefits With Anti-EGFR Agents} Because there was significant heterogeneity between the studies ( $\left.P_{\text {heterogeneity }}=.094 ; I^{2}=53.4 \%\right)$ in the left-sided subgroup, a random-effects model was used for the PFS analysis. The pooled HRs of 0.70 (95\% CI, 0.57-0.86; $P=.001$ ) showed that patients with left-sided $R A S \mathrm{wt}$ mCRC treated with chemotherapy + Cet/Pani had a significantly higher PFS compared with those treated with chemotherapy alone (Figure 2B). Unlike OS, a significant improvement in PFS with Cet/Pani was detected in patients with $R A S$ wt right-sided tumors (HR, 0.76; 95\% CI, 0.59-0.99; $P=.040$; Figure 2B), absent betweenstudy heterogeneity $\left(P_{\text {heterogeneity }}=.926 ; I^{2}=0 \%\right)$. Multivariate meta-regression analysis revealed no significant 


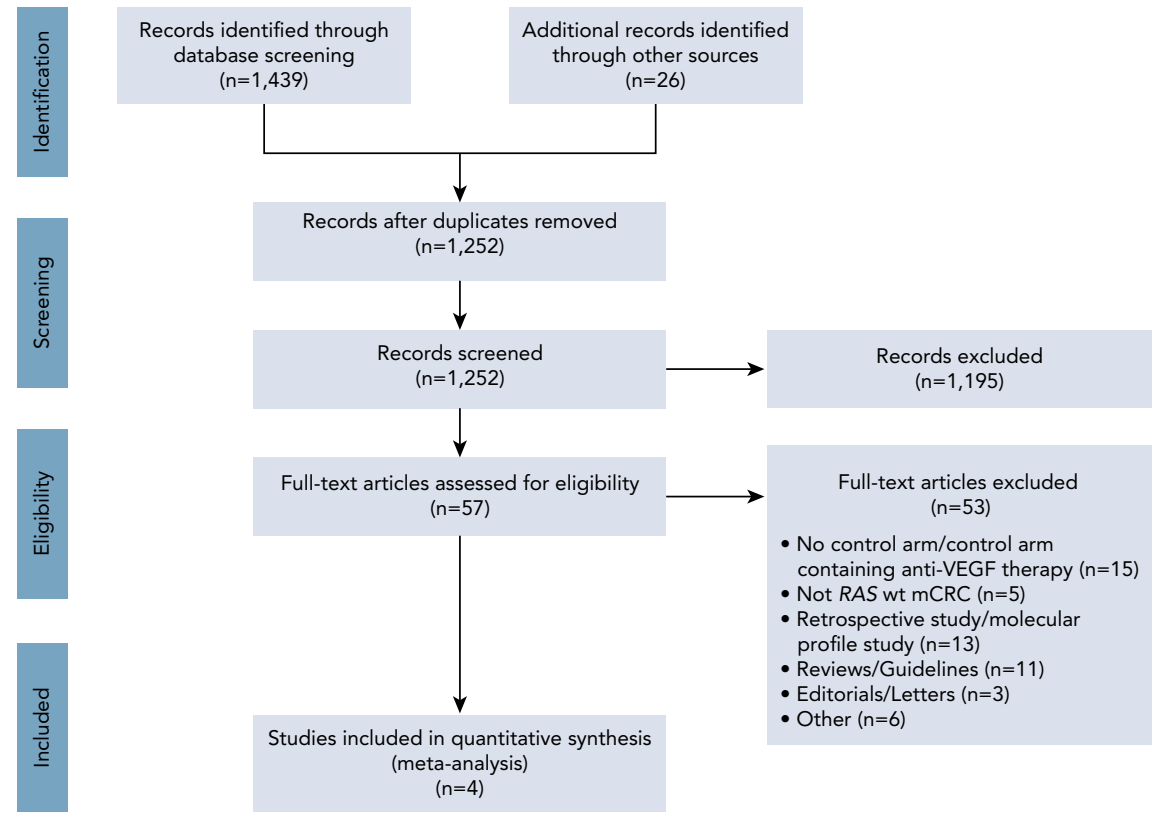

Figure 1. Search results summarized according to PRISMA guidelines.

Abbreviations: $\mathrm{mCRC}$, metastatic colorectal cancer; VEGF, vascular endothelial growth factor; wt, wild-type.

interaction between PTL and the efficacy of Cet/Pani on PFS $\left(P_{\text {interaction }}=.591\right.$; Table 2$)$.

\section{Effect of PTL on ORR Benefits With Anti-EGFR Agents} The addition of Cet/Pani to chemotherapy significantly improved ORR in both patients with $R A S$ wt left-sided tumors (OR, 3.28; 95\% CI, 1.95-5.51; $P<.0001 ; P_{\text {heterogeneity }}=$ $\left..002 ; I^{2}=80.1 \%\right)$ and those with $R A S$ wt right-sided tumors (OR, 1.78; 95\% CI, 1.08-2.93; $P=.024 ; P_{\text {heterogeneity }}=.527$; $I^{2}=0 \%$; Figure 2C). Multivariate meta-regression analysis indicated no significant interaction between PTL and the treatment effect of Cet/Pani on ORR $\left(P_{\text {interaction }}=.134\right.$; Table 2).

Because treatment line was a significant predictor of the treatment effect of Cet/Pani on ORR $\left(P_{\text {interaction }}=.024\right.$; Table 2), we performed a pooled analysis of ORR based on the 3 first-line RCTs. ORRs in patients with RAS wt left-sided tumors were $68.9 \%$ (314 of 456) in those treated with chemotherapy + Cet/Pani and 45.6\% (208 of 456) in those treated with chemotherapy alone $(P<.0001)$. In patients with $R A S$ wt right-sided tumors, 50 of 116 (43.1\%) treated with chemotherapy + Cet/Pani achieved an objective response compared with 42 of 135 (31.1\%) treated with chemotherapy alone $(P=.001)$.

\section{Discussion}

Previous studies have reported differences in the efficacy of anti-EGFR agents in RAS wt mCRC according to PTL. ${ }^{4,8}$ Notably, 2 meta-analyses including the CALGB 80405, FIRE-3, and PEAK studies showed significantly better OS, PFS, and ORR with first-line chemotherapy plus anti-EGFR antibodies than with chemotherapy $+\mathrm{Bev}$ in patients with $R A S$ wt left-sided mCRC. ${ }^{10,17}$ In contrast, patients with $R A S$ wt right-sided tumors appeared to gain more benefits from chemotherapy + Bev. ${ }^{10,17}$ These data led the NCCN Clinical Practice Guidelines in Oncology

\section{Table 1. Baseline Characteristics of Included Trials}

\begin{tabular}{|c|c|c|c|c|c|c|c|}
\hline Trial & Phase & Study Population & $\begin{array}{c}\text { Included } \\
\text { Patients, }{ }^{\text {a }} \text { (\%) }\end{array}$ & $\begin{array}{c}\text { Patients With Right-Sided } \\
\text { Tumors, }^{\text {b }} \text { n (\%) }\end{array}$ & $\begin{array}{l}\text { Chemotherapy } \\
\text { Backbone }\end{array}$ & $\begin{array}{l}\text { Anti-EGFR } \\
\text { Agent }\end{array}$ & $\begin{array}{l}\text { Treatment } \\
\text { Line }\end{array}$ \\
\hline CRYSTAL 8,21 & III & All RAS wt and tumor side confirmed & $364(29.9)$ & $84(23.1)$ & FOLFIRI & Cetuximab & First \\
\hline PRIME $^{11,22}$ & III & All RAS wt and tumor side confirmed & $416(35.2)$ & $88(21.2)$ & FOLFOX & Panitumumab & First \\
\hline TAILOR $^{19}$ & III & All RAS wt and tumor side confirmed & $391(77.6)$ & $83(21.2)$ & FOLFOX & Cetuximab & First \\
\hline $20050181^{23,24}$ & III & All RAS wt and tumor side confirmed & $368(31.0)$ & $70(19.0)$ & FOLFIRI & Panitumumab & Second \\
\hline
\end{tabular}

Abbreviations: EGFR, epidermal growth factor receptor; FOLFIRI, fluorouracil/leucovorin/irinotecan; FOLFOX, fluorouracil/leucovorin/oxaliplatin; wt, wild-type. apatients in the study population and its percentage in the all-randomized population.

bIncluded patients with right-sided tumors and its percentage in the study population. In all 4 included trials, primary tumors originating in the cecum, appendix, ascending colon, hepatic flexure, and transverse colon were classified as right-sided. 
A

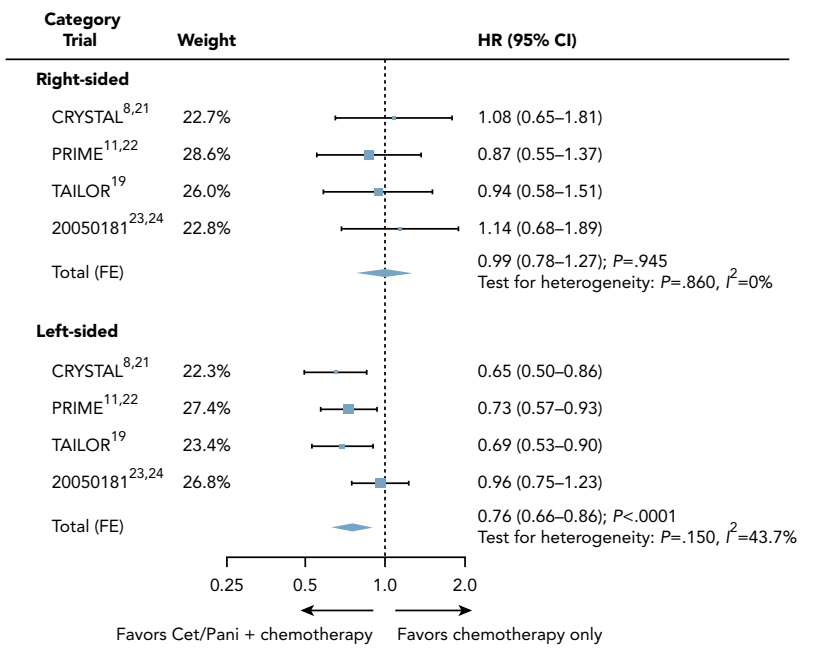

C

\begin{tabular}{|c|c|c|c|}
\hline $\begin{array}{l}\text { Category } \\
\text { Trial }\end{array}$ & Weight & & OR $(95 \% \mathrm{Cl})$ \\
\hline \multicolumn{4}{|l|}{ Right-sided } \\
\hline CRYSTAL ${ }^{8,21}$ & $29.7 \%$ & $\longrightarrow$ & $1.45(0.58-3.64)$ \\
\hline PRIME $^{11,22}$ & $37.5 \%$ & & $1.36(0.60-3.08)$ \\
\hline TAILOR $^{19}$ & $27.8 \%$ & $\longrightarrow$ & $2.58(1.00-6.67)$ \\
\hline $20050181^{23,24}$ & $5.0 \%$ & $\longrightarrow$ & $5.69(0.60-53.63)$ \\
\hline Total (FE) & & - & $\begin{array}{l}1.78(1.08-2.93) ; P=.024 \\
\text { Test for heterogeneity: } P=.527, I^{2}=0 \%\end{array}$ \\
\hline \multicolumn{4}{|l|}{ Left-sided } \\
\hline CRYSTAL $^{8,21}$ & $24.2 \%$ & 一 & $3.99(2.40-6.62)$ \\
\hline PRIME $^{11,22}$ & $27.4 \%$ & $-\square$ & $1.91(1.33-2.72)$ \\
\hline TAILOR $^{19}$ & $25.2 \%$ & - & $2.60(1.64-4.14)$ \\
\hline $20050181^{23,24}$ & $23.2 \%$ & $\longrightarrow$ & $6.49(3.73-11.30)$ \\
\hline Total (RE) & & - & $\begin{array}{l}3.28(1.95-5.51) ; P<.0001 \\
\text { Test for heterogeneity: } P=.002, I^{2}=80.1 \%\end{array}$ \\
\hline \multicolumn{4}{|c|}{ 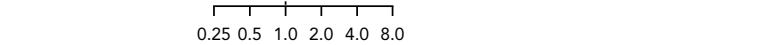 } \\
\hline
\end{tabular}

B

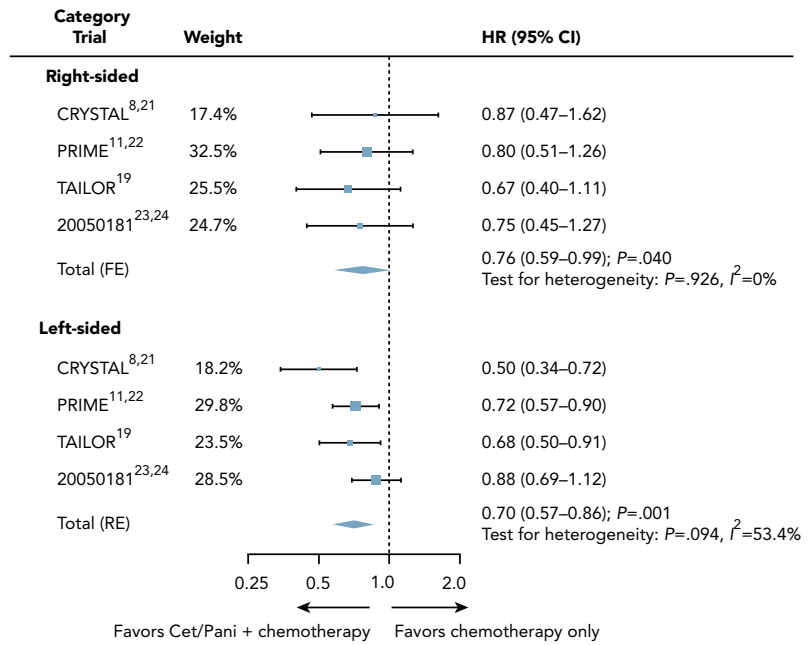

Figure 2. Forest plots showing the HRs for (A) overall survival and (B) progression-free survival and the odds ratio for (C) objective response rate comparing chemotherapy + Cet or Pani with chemotherapy only, with stratification for tumor location. Interaction test between tumor side and treatment effect were (A) $P=.044$, (B) $P=.591$, and (C) $P=.134$.

Abbreviations: Cet, cetuximab; FE, fixed-effects model; HR, hazard ratio; Pani, panitumumab; RE, random-effects model.

(NCCN Guidelines) for Colon Cancer to recently recommend against use of anti-EGFR agents in the frontline treatment of $R A S$ wt right-sided mCRC. ${ }^{18}$

Our updated meta-analysis confirmed that adding Cet/Pani to chemotherapy clearly benefited patients with RAS wt left-sided tumors in terms of OS, PFS, and ORR. In contrast, the treatment effect of Cet/Pani on OS was significantly attenuated in the RAS wt rightsided subgroup, which corroborates previous observations that have convincingly shown fewer benefits from anti-EGFR therapy in patients with right-sided tumors. ${ }^{4,8,25}$ To the best of our knowledge, our study is the first to show that the addition of Cet/Pani to chemotherapy could still significantly improve PFS and ORR in patients with right-sided $R A S$ wt mCRC. In previous meta-analyses, we noticed a numerical trend toward better PFS and ORR favoring chemotherapy + Cet/Pani over chemotherapy alone, but results were not statistically significant. ${ }^{10,17}$ A possible reason for our different findings might be the additional inclusion of the TAILOR trial, which provided a greater statistical power from the increased sample size of patients with $R A S$ wt right-sided tumors, absent between-study heterogeneity.

The improved ORR (from $31 \%$ to $43 \%$ in the first line) with chemotherapy + Cet/Pani versus chemotherapy alone suggests that anti-EGFR agents are 


\begin{tabular}{|c|c|c|c|c|c|c|}
\hline Variable & $\mathrm{HR}_{\text {interaction }}(95 \% \mathrm{Cl})$ & $P_{\text {interaction }}{ }^{a}$ & $\mathrm{HR}_{\text {interaction }}(95 \% \mathrm{Cl})$ & $P_{\text {interaction }}$ & $\mathrm{OR}_{\text {interaction }}(95 \% \mathrm{Cl})$ & $P_{\text {interaction }}{ }^{\mathrm{a}}$ \\
\hline \multicolumn{7}{|l|}{ Tumor side } \\
\hline Left vs right & $0.75(0.57-0.99)$ & .044 & $0.92(0.69-1.24)$ & .591 & $1.53(0.88-2.66)$ & .134 \\
\hline \multicolumn{7}{|l|}{ Anti-EGFR agent } \\
\hline \multicolumn{7}{|l|}{ Chemotherapy backbone } \\
\hline FOLFOX vs FOLFIRI & $1.02(0.73-1.42)$ & .922 & $1.17(0.77-1.76)$ & .458 & $0.81(0.44-1.49)$ & .490 \\
\hline \multicolumn{7}{|l|}{ Treatment line } \\
\hline First- vs second-line & $1.34(0.85-2.11)$ & .206 & $1.36(0.82-2.27)$ & .235 & $2.76(1.15-6.65)$ & .024 \\
\hline
\end{tabular}

Abbreviations: Cet, cetuximab; EGFR, epidermal growth factor receptor; FOLFIRI, fluorouracil/leucovorin/irinotecan; FOLFOX, fluorouracil/leucovorin/oxaliplatin; $\mathrm{HR}$, hazard ratio; Pani, panitumumab.

aBolded $P$ values indicate statistical significance.

worthy of consideration for patients with $R A S$ wt rightsided tumors when cytoreduction is set as the therapeutic goal. A previous study showed a strong correlation between ORR and the R0 resection rate among patients with initially unresectable liver metastases ${ }^{26}$ Notwithstanding that right-sided tumors have a more dismal prognosis than left-sided tumors, several studies have reported favorable survival outcomes after curative-intent hepatectomy even in patients with right-sided tumors (median OS $>3$ years), ${ }^{27,28}$ suggesting that these patients may also substantially benefit from improvements in the rate of conversion to resectability. Recent subgroup analysis data from the TRIBE study revealed significantly improved ORR and PFS, and OS favoring the triplet chemotherapy (fluorouracil/oxaliplatin/ irinotecan [FOLFOXIRI]) + Bev versus FOLFIRI + Bev in patients with right-sided mCRC. ${ }^{29}$ The ORR was as high as $63.9 \%$ in right-sided tumors and reached $81.3 \%$ among RAS/ BRAF wt right-sided tumors. ${ }^{29}$ Therefore, in patients with right-sided tumors for whom cytoreduction is the goal, FOLFOXIRI + Bev would be the preferred therapeutic regimen when they fulfill the clinical criteria for use of FOLFOXIRI (ie, age 18-75 years and performance status $0-2$ if age $\leq 70$ years, or 0 if age $71-75$ years).$^{30} \mathrm{In}$ prior meta-analyses of the CALGB 80405, FIRE-3, and PEAK studies, chemotherapy doublets plus anti-EGFR agents exhibited a numerically superior ORR to chemotherapy doublets plus $\mathrm{Bev},{ }^{10,17}$ whereas no trials have compared chemotherapy doublets plus anti-EGFR agents with FOLFOXIRI alone head-to-head. In view of the increased toxicity of FOLFOXIRI compared with chemotherapy doublets ${ }^{31}$ and the different toxicity profiles between Bev and anti-EGFR agents, ${ }^{32-34}$ a chemotherapy doublet plus anti-EGFR antibody would still be a reasonable option for patients with $R A S$ wt rightsided tumors when they need significant tumor response but are not candidates for either FOLFOXIRI or Bev therapy.
Currently, the role of anti-EGFR therapy in patients with right-sided mCRC seems limited when the treatment goal is prolongation of disease control. Prior meta-analyses of the 3 first-line trials comparing chemotherapy plus anti-EGFR agents versus chemotherapy + Bev revealed significantly better PFS and numerically better OS favoring chemotherapy $+\mathrm{Bev}$ in patients with $R A S$ wt right-sided mCRC. ${ }^{10,17}$ This finding led to increasing preference for using Bev as the targeted agent in initial therapy for right-sided tumors. ${ }^{17}$ Nonetheless, the choice between chemotherapy plus anti-EGFR agents and chemotherapy alone is still clinically relevant to patients with $R A S$ wt right-sided mCRC when they have contraindications to Bev therapy. Notably, our study found that the addition of Cet/Pani to chemotherapy significantly improved PFS in patients with RAS wt right-sided mCRC, which suggests the non-negligible activity of anti-EGFR agents in disease stabilization for these patients. In view of the rather dismal prognosis of patients with right-sided mCRC, ${ }^{3,25}$ prolongation of disease stabilization could potentially delay fitness deterioration and offer them a better chance to be included in future clinical trials, even though improvement in OS was not shown. Thus, a chemotherapy doublet in combination with anti-EGFR antibody could be considered for patients with $R A S$ wt right-sided tumors when disease stabilization is the goal and Bev therapy is not feasible.

We acknowledge certain limitations in our analysis. First, this meta-analysis was performed at the study level rather than the individual patient level. Thus, some of the unreported information at the individual patient level, such as $B R A F$ status, might affect our findings. The prevalence of $B R A F$ mutation with stratification for PTL was reported in the PRIME, 20050181, and TAILOR studies and was generally balanced between treatment arms across these studies. ${ }^{17,19}$ The efficacy data of anti-EGFR therapy with stratification for both $B R A F$ status and 
PTL was only available in the TAILOR study, which showed that OS and PFS were even worse with antiEGFR therapy for $R A S \mathrm{wt} / B R A F$-mutant right-sided tumors, but seemed to favor anti-EGFR therapy for $R A S / B R A F$ wt right-sided tumors. ${ }^{19}$ This finding, which corroborates previous observations, ${ }^{35,36}$ suggests that anti-EGFR agents are highly unlikely to yield benefits in $R A S$ wt / BRAF-mutant right-sided tumors. Therefore, for patients with right-sided tumors, it could be justified to confine anti-EGFR therapy to those with $R A S / B R A F$ wt tumors.

Second, our meta-analysis was based on post hoc subset analysis data regarding either historically examined $R A S$ status or retrospectively collected PTL information. Consequently, a substantial proportion of patients who had a KRAS/NRAS mutation, nonevaluable $R A S$ status, or missing data regarding PTL were excluded from the all-randomized population. Misclassification of tumor sidedness might also have occurred during retrospective data collection. Therefore, our findings may experience potential biases and should be interpreted with caution. However, given that no available trial prospectively examines the impact of PTL on efficacy of anti-EGFR therapy in $R A S$ wt mCRC, our meta-analysis still presents reasonably compelling evidence regarding this issue.

Third, the 4 included trials were different in study design; however, there was no heterogeneity among the studies within the right-sided subgroup for all 3 end points, suggesting the robustness of the pooled analysis data for right-sided tumors.

\section{Conclusions}

Given the ORR and PFS benefits of chemotherapy + Cet/ Pani versus chemotherapy alone, anti-EGFR therapies should not be excluded from treatment of RAS wt right-sided mCRC. A chemotherapy doublet plus antiEGFR antibody remains an option for patients with $R A S$ wt right-sided tumors when Bev therapy is not feasible, independent of treatment goal; for those in need of cytoreduction but ineligible for FOLFOXIRI, chemotherapy doublets with anti-EGFR agents could also be considered.

\section{Acknowledgments}

The authors wish to thank Dr. A.H. Lin (Department of Medical Statistics and Epidemiology, School of Public Health, Sun Yat-sen University) for suggestions and assistance in data analysis.

Submitted June 25, 2018; accepted for publication January 28, 2019.

Author contributions: Study concept and design: Z.X. Wang, Wu, Xu. Literature retrieval: Z.X. Wang, Wu. Data acquisition \& risk of bias assessment: Z.X. Wang, Wu, He, Luo, Ding, Xie, Chen, Li, F. Wang, Xu. Data analysis and interpretation: Z.X. Wang, Wu, He, Y.N. Wang, Xu. Manuscript preparation: Z.X. Wang, Wu. Critical revisions: All authors.

Disclosures: The authors have disclosed that they have not received any financial considerations from any person or organization to support the preparation, analysis, results, or discussion of this article.

Funding: This study was supported by grants from the Natural Science Foundation of Guangdong Province (2014A030312015), Science and Technology Program of Guangdong (2015B020232008), and Science and Technology Program of Guangzhou (15570006, 201508020250, 201604020003).

Correspondence: Rui-Hua Xu, MD, PhD, Department of Medical Oncology Sun Yat-sen University Cancer Center, State Key Laboratory of Oncology in South China, Collaborative Innovation Center for Cancer Medicine, 651 Dong Feng Road East, Guangzhou 510060, China. Email: xurh@sysucc.org.cn

\section{References}

1. Pourhoseingholi MA, Vahedi M, Baghestani AR. Burden of gastrointestinal cancer in Asia; an overview. Gastroenterol Hepatol Bed Bench 2015;8: 19-27

2. Schrag D, Weng S, Brooks G, et al. The relationship between primary tumor sidedness and prognosis in colorectal cancer [abstract]. J Clin Oncol 2016;34(Suppl):Abstract 3505.

3. Petrelli F, Tomasello G, Borgonovo K, et al. Prognostic survival associated with left-sided vs right-sided colon cancer: a systematic review and metaanalysis. JAMA Oncol 2017;3:211-219.

4. Venook AP, Niedzwiecki D, Innocenti $F$, et al. Impact of primary $\left(1^{\circ}\right)$ tumor location on overall survival (OS) and progression-free survival (PFS) in patients (pts) with metastatic colorectal cancer (mCRC): analysis of CALGB/SWOG 80405 (Alliance) [abstract]. J Clin Oncol 2016;34(Suppl): Abstract 3504

5. Maus MK, Hanna DL, Stephens C, et al. Gene expression profiles and tumor locations in colorectal cancer (left vs right vs rectum) [abstract] J Clin Oncol 2013;31(Suppl):Abstract 3527.

6. Missiaglia E, Jacobs B, D'Ario G, et al. Distal and proximal colon cancers differ in terms of molecular, pathological, and clinical features. Ann Oncol 2014;25:1995-2001

7. Meza R, Jeon J, Renehan AG, et al. Colorectal cancer incidence trends in the United States and United kingdom: evidence of right- to left-sided biological gradients with implications for screening. Cancer Res 2010;70: 5419-5429.

8. Tejpar S, Stintzing S, Ciardiello F, et al. Prognostic and predictive relevance of primary tumor location in patients with RAS wild-type metastatic colorectal cancer: retrospective analyses of the CRYSTAL and FIRE-3 trials. JAMA Oncol 2017;3:194-201.

9. Benedix F, Kube R, Meyer F, et al. Comparison of 17,641 patients with rightand left-sided colon cancer: differences in epidemiology, perioperative course, histology, and survival. Dis Colon Rectum 2010;53:57-64.

10. Holch JW, Ricard I, Stintzing S, et al. The relevance of primary tumour location in patients with metastatic colorectal cancer: a meta-analysis of first-line clinical trials. Eur J Cancer 2017;70:87-98.

11. Boeckx N, Koukakis R, Op de Beeck K, et al. Primary tumor sidedness has an impact on prognosis and treatment outcome in metastatic colorectal cancer: results from two randomized first-line panitumumab studies. Ann Oncol 2017;28:1862-1868.

12. Wang F, Bai L, Liu TS, et al. Right-sided colon cancer and left-sided colorectal cancers respond differently to cetuximab. Chin J Cancer 2015; 34:384-393.

13. He WZ, Liao FX, Jiang $C$, et al. Primary tumor location as a predictive factor for first-line bevacizumab effectiveness in metastatic colorectal cancer patients. J Cancer 2017;8:388-394.

14. Moretto R, Cremolini C, Rossini D, et al. Location of primary tumor and benefit from anti-epidermal growth factor receptor monoclonal antibodies in patients with RAS and BRAF wild-type metastatic colorectal cancer. Oncologist 2016;21:988-994.

15. Boisen MK, Johansen JS, Dehlendorff C, et al. Primary tumor location and bevacizumab effectiveness in patients with metastatic colorectal cancer Ann Oncol 2013;24:2554-2559.

16. Heinemann V, Modest DP, von Weikersthal LF, et al. Gender and tumor location as predictors for efficacy: influence on endpoints in first-line 
treatment with FOLFIRI in combination with cetuximab or bevacizumab in the AIO KRK 0306 (FIRE3) trial [abstract]. J Clin Oncol 2014;32(Suppl): Abstract 3600.

17. Arnold D, Lueza B, Douillard JY, et al. Prognostic and predictive value of primary tumour side in patients with RAS wild-type metastatic colorectal cancer treated with chemotherapy and EGFR directed antibodies in six randomized trials. Ann Oncol 2017;28:1713-1729.

18. Benson AB, Venook AP, Bekaii-Saab T, et al. NCCN Clinical Practice Guidelines in Oncology: Colon Cancer. Version 1.2017. Accessed June 15,2017 . To view the most recent version, visit NCCN.org.

19. Qin S, Li J, Wang L, et al. Efficacy and tolerability of first-line cetuximab plus leucovorin, fluorouracil, and oxaliplatin (FOLFOX-4) versus FOLFOX-4 in patients with RAS wild-type metastatic colorectal cancer: the open-label, randomized, phase III TAILOR trial [published online September 10, 2018]. J Clin Oncol, doi: 10.1200/JCO.2018.78.3183

20. Liberati A, Altman DG, Tetzlaff J, et al. The PRISMA statement for reporting systematic reviews and meta-analyses of studies that evaluate healthcare interventions: explanation and elaboration. BMJ 2009;339: b2700.

21. Van Cutsem E, Köhne CH, Hitre E, et al. Cetuximab and chemotherapy as initial treatment for metastatic colorectal cancer. N Engl J Med 2009;360: 1408-1417.

22. Douillard JY, Siena S, Cassidy J, et al. Randomized, phase III trial of panitumumab with infusional fluorouracil, leucovorin, and oxaliplatin (FOLFOX4) versus FOLFOX4 alone as first-line treatment in patients with previously untreated metastatic colorectal cancer: the PRIME study. J Clin Oncol 2010;28:4697-4705.

23. Peeters M, Price TJ, Cervantes A, et al. Randomized phase III study of panitumumab with fluorouracil, leucovorin, and irinotecan (FOLFIRI) compared with FOLFIRI alone as second-line treatment in patients with metastatic colorectal cancer. J Clin Oncol 2010;28:4706-4713.

24. Boeckx N, Koukakis R, Op de Beeck K, et al. Effect of primary tumor location on second- or later-line treatment outcomes in patients with RAS wild-type metastatic colorectal cancer and all treatment lines in patients with RAS mutations in four randomized panitumumab studies. Clin Colorectal Cancer 2018;17:170-178.

25. Brulé SY, Jonker DJ, Karapetis CS, et al. Location of colon cancer (rightsided versus left-sided) as a prognostic factor and a predictor of benefit from cetuximab in NCIC CO.17. Eur J Cancer 2015;51:1405-1414.

26. Okuno $M$, Hatano $E$, Nishino $H$, et al. Does response rate of chemotherapy with molecular target agents correlate with the conversion rate and survival in patients with unresectable colorectal liver metastases?: a systematic review. Eur J Surg Oncol 2017;43:1003-1012.
27. Sasaki K, Andreatos N, Margonis GA, et al. The prognostic implications of primary colorectal tumor location on recurrence and overall survival in patients undergoing resection for colorectal liver metastasis. J Surg Oncol 2016;114:803-809.

28. Creasy JM, Sadot E, Koerkamp BG, et al. The impact of primary tumor location on long-term survival in patients undergoing hepatic resection for metastatic colon cancer. Ann Surg Oncol 2018;25:431-438.

29. Cremolini $\mathrm{C}$, Antoniotti $\mathrm{C}$, Lonardi $\mathrm{S}$, et al. Primary tumor sidedness and benefit from FOLFOXIRI plus bevacizumab as initial therapy for metastatic colorectal cancer. Retrospective analysis of the TRIBE trial by GONO [published online April 20, 2018]. Ann Oncol, doi: 10.1093/annonc/ mdy 140

30. Falcone A, Ricci S, Brunetti I, et al. Phase III trial of infusional fluorouracil, leucovorin, oxaliplatin, and irinotecan (FOLFOXIRI) compared with infusional fluorouracil, leucovorin, and irinotecan (FOLFIRI) as first-line treatment for metastatic colorectal cancer: the Gruppo Oncologico Nord Ovest. J Clin Oncol 2007;25:1670-1676.

31. Marques RP, Duarte GS, Sterrantino C, et al. Triplet (FOLFOXIRI) versus doublet (FOLFOX or FOLFIRI) backbone chemotherapy as first-line treatment of metastatic colorectal cancer: a systematic review and metaanalysis. Crit Rev Oncol Hematol 2017;118:54-62.

32. Schwartzberg LS, Rivera F, Karthaus M, et al. PEAK: a randomized, multicenter phase II study of panitumumab plus modified fluorouracil, leucovorin, and oxaliplatin (mFOLFOX6) or bevacizumab plus mFOLFOX6 in patients with previously untreated, unresectable, wild-type KRAS exon 2 metastatic colorectal cancer. J Clin Oncol 2014;32:2240-2247.

33. Venook AP, Niedzwiecki D, Lenz HJ, et al. Effect of first-line chemotherapy combined with cetuximab or bevacizumab on overall survival in patients with KRAS wild-type advanced or metastatic colorectal cancer: a randomized clinical trial. JAMA 2017;317:2392-2401.

34. Heinemann $V$, von Weikersthal LF, Decker $T$, et al. FOLFIRI plus cetuximab versus FOLFIRI plus bevacizumab as first-line treatment for patients with metastatic colorectal cancer (FIRE-3): a randomised, open-label, phase 3 trial. Lancet Oncol 2014;15:1065-1075.

35. Rowland A, Dias MM, Wiese MD, et al. Meta-analysis of BRAF mutation as a predictive biomarker of benefit from anti-EGFR monoclonal antibody therapy for RAS wild-type metastatic colorectal cancer. Br J Cancer 2015 112:1888-1894.

36. Pietrantonio F, Petrelli F, Coinu A, et al. Predictive role of BRAF mutations in patients with advanced colorectal cancer receiving cetuximab and panitumumab: a meta-analysis. Eur J Cancer 2015; 51:587-594 